\title{
Gluon propagator in an external field; what happens when the field is removed?
}

\author{
Axel Maas * \\ Friedrich-Schiller-University Jena \\ E-mail: axelmaas@web.de
}

\section{Daniel Zwanziger ${ }^{\dagger}$}

New York University

E-mail: daniel.zwanzigerenyu.edu

\begin{abstract}
We exhibit some general bounds on the free energy $W(J)$ in an $\mathrm{SU}(\mathrm{N})$ gauge theory, where $J_{\mu}^{b}$ is a source for the gluon field $A_{\mu}^{b}$ in the minimal Landau gauge, and $W(J)$ is the generating functional of connected correlators, $\exp W(J)=\langle\exp (J, A)\rangle$. We then specialize to a source $J(x)=h \cos (k \cdot x)$ of definite momentum $k$ and source strength $h$, and study the gluon propagator $D(k, h)$ in the presence of this source. Among other relations, we prove $\int_{0}^{\infty} d h D(k, h) \leq \sqrt{2} k$, which implies $\lim _{k \rightarrow 0} D(k, h)=0$, for all positive $h>0$. This means that the system does not respond to a static color probe, no matter how strong.

We also present numerical evaluations of the free energy $W(k, h)$ and the gluon propagator $D(k, h)$ for the case of SU(2) Yang-Mills theory in dimensions 2, 3 and 4 which are consistent with these findings, and we compare with recent lattice calculations at $h=0$ which indicate that the gluon propagator in the minimum Landau gauge is finite, $\lim _{k \rightarrow 0} D(k, 0)>0$. These lattice data together with our analytic results imply a jump in the value of $D(k, h)$ at $h=0$ and $k=0$, and the value of $D(k, h)$ at this point depends on the order of limits.
\end{abstract}

From quarks and gluons to hadronic matter: A bridge too far?

2-6 September, 2013

European Centre for Theoretical Studies in Nuclear Physics and Related Areas (ECT*), Villazzano, Trento (Italy)

\footnotetext{
*Supported by the DFG under grant numbr MA3935/5-1

† Speaker.
} 


\section{Introduction}

We shall be concerned with the Euclidean correlators of gluons in QCD with an $S U(N)$ local gauge symmetry that are fixed to the minimal Landau gauge. This gauge is obtained by minimizing the Hilbert square norm $[1,2,3]$

$$
F_{A}(g) \equiv\left\|{ }^{g} A\right\|^{2} ; \quad\|A\|^{2} \equiv \int\left|A_{\mu}^{b}(x)\right|^{2} d^{4} x,
$$

to some local minimum (in general not an absolute minimum) with respect to local gauge transformations $g(x)$. These act according to

$$
{ }^{g} A_{\mu}=g^{-1} A_{\mu} g+g^{-1} \partial_{\mu} g .
$$

At a local minimum, the functional $F_{A}(g)$ is stationary and its second variation is positive. As is well known, these two properties imply respectively that the Landau gauge (transversality) condition is satisfied and that the Faddeev-Popov operator is positive, that is,

$$
\partial \cdot A=0 ; \quad(\omega, M(A) \omega) \geq 0
$$

for all $\omega$. Here the Faddeev-Popov operator acts according to

$$
M^{a c}(A) \omega^{c}=-\partial_{\mu} D_{\mu}^{a c}(A) \omega^{c},
$$

where the gauge covariant derivative is defined by

$$
D_{\mu}^{a c}(A) \omega^{c}=\partial_{\mu} \omega^{a}+f^{a b c} A_{\mu}^{b} \omega^{c} .
$$

Configurations $A$ that satisfy these two conditions are said to be in the (first) Gribov region [4] which we designate by $\Omega$. In general there are more than one local minimum of $F_{A}(g)$, and we do not specify which local minimum is achieved. This gauge is realized numerically by minimizing a lattice analog of $F_{A}(g)$ by some algorithm, and the local minimum achieved is in general algorithm dependent.

The analytic bounds which we shall obtain follow from the restriction of the gauge-fixed configurations to the Gribov region $\Omega$, and are the same whether the gluons are coupled to quarks as in full QCD, or not, as in pure gluodynamics. In fact the same bounds hold for any gauge bosons with $S U(N)$ gauge symmetry, for example, with coupling to a Higgs field [5], provided only that they are in the minimal Landau gauge.

\section{General bounds on free energy}

In the minimal Landau gauge, the free energy $W(J)$ is defined by

$$
\exp W(J) \equiv \int_{\Omega} d A \rho(A) \exp (J, A) .
$$

Here the quark degrees of freedom (if present) are integrated out. The Euclidean probability $\rho(A)$ includes the Yang-Mills action, the gauge-fixing factor $\delta(\partial \cdot A)$, the Faddeev-Popov determinant, 
and possibly the quark determinant. We shall use only the properties $\rho(A) \geq 0$ and $\int d A \rho(A)=1$. The source term $J_{\mu}^{a}(x)$ is real and is taken to be transverse $\partial \cdot J=0$ without loss of generality because $A$ is identically transverse. The free energy per unit Euclidean volume, $w(J)=W(J) / V$, is the generating functional of connected correlators,

$$
\langle A(x) A(y) \ldots\rangle_{\mathrm{conn}}=\left.\frac{\delta}{\delta J_{x}} \frac{\delta}{\delta J_{y}} \ldots w(J)\right|_{J=0} .
$$

The general bound is immediate. From the inequality $(J, A) \leq \max _{A \in \Omega}(J, A)=\left(J, A_{\max }\right)$, where $A_{\max }$ is that configuration in $\Omega$ that maximizes $(J, A)$ for fixed $J$, we obtain

$$
\begin{aligned}
\exp W(J) & \leq \int_{\Omega} d A \rho(A) \exp \left(J, A_{\max }\right) \\
& =\exp \left(J, A_{\max }\right)
\end{aligned}
$$

which gives the bound

$$
W(J) \leq \max _{A \in \Omega}(J, A) .
$$

Because $\Omega$ is bounded in every direction [6], this bound is finite. It is not hard to show that the maximum occurs when $A$ lies on the boundary $\partial \Omega$ of $\Omega$, and we have the more precise bound on the free energy [7],

$$
W(J) \leq \max _{A \in \partial \Omega}(J, A) .
$$

The right hand side is linear in $J$,

$$
\max _{A \in \partial \Omega}(h J, A)=h \max _{A \in \partial \Omega}(J, A)
$$

for $h>0$. This linear bound is a characteristic feature for integration over any bounded region, such as the Gribov region. By contrast, for a free field the free energy is quadratic in $J, W_{\text {free }}(J)=$ $(1 / 2)\left(J, K^{-1} J\right)$, where $K=-\partial^{2}+m^{2}$, which strongly violates the linear bound (2.5) at large $J$.

\section{Bound for a plane-wave source}

We now specialize to a plane wave source,

$$
J_{\mu}^{a}(x)=h \cos \left(k \cdot x_{2}\right) \delta^{a 3} \delta_{\mu 1},
$$

Here $h$ is the analog in a spin theory of an external magnetic field, modulated by a plane wave. The wave number takes on the values $k=2 \pi n / L$, where $n$ is an integer, and $L$ is the edge of a periodic Euclidean box. The indices 1 and 2 are chosen so $J$ is transverse, $\partial_{\mu} J_{\mu}=0$. For this source, we parametrize the free energy per unit Euclidean volume $V=L^{d}$ by

$$
w(k, h) \equiv W(h \cos (k \cdot x)) / V .
$$

The gluon propagator is its second derivative at $h=0$,

$$
D(k)=\left.(1 / 2) \frac{\partial^{2} w(k, h)}{\partial h^{2}}\right|_{h=0} .
$$


For a static source, $k=0$, we have $A_{\max }(x)=$ const, and the Faddeev-Popov operator may be diagonalized by Fourier transform. In this case we obtain on a finite volume $V=L^{d}$, the bound [8]

$$
w(0, h) \leq|h|(2 \pi / L) .
$$

Thus on an infinite volume, $L \rightarrow \infty$, the free energy vanishes,

$$
\lim _{L \rightarrow \infty} w(0, h)=0
$$

for a static source of strength $h$ no matter how strong. Lest it be thought that this is a peculiarity of the mode $k=0$, we also exhibit a bound at infinite volume, with $k$ finite. By an explicit calculation one obtains [8]

$$
w(k, h) \leq|h k| .
$$

The free energy vanishes in the static limit

$$
\lim _{k \rightarrow 0} w(k, h)=0
$$

for all $h$, no matter how strong.

We also obtain a bound on the "magnetization" $m(k, h) \equiv \frac{\partial w(k, h)}{\partial h}$. Indeed because $\rho(A)$ is normalized, we have $w(k, 0)=0$, and so

$$
w(k, h)=\int_{0}^{h} d h^{\prime} m\left(k, h^{\prime}\right) .
$$

Inserting this into the bound (3.6), we obtain, for $k>0$ and $h>0$,

$$
\frac{1}{h} \int_{0}^{h} d h^{\prime} m\left(k, h^{\prime}\right) \leq k
$$

which gives

$$
\lim _{k \rightarrow 0} \frac{1}{h} \int_{0}^{h} d h^{\prime} m\left(k, h^{\prime}\right)=0 .
$$

Note that $m(k, 0)=0$ because there is no spontaneous magnetization, and that $\frac{\partial m(k, h)}{\partial h}=\frac{\partial^{2} w(k, h)}{\partial h^{2}} \geq$ 0 is positive because $w(k, h)$ is a convex function of $h$. Consequently $m(k, h)$ is nonnegative, $m(k, h) \geq 0$, and we conclude from the positivity of the integrand and the vanishing of the last integral that the magnetization produced by a static source vanishes,

$$
\lim _{k \rightarrow 0} m(k, h)=0
$$

for almost all $h$. Thus, in the minimal Landau gauge, the static static color degree of freedom cannot be excited by applying an external color-magnetic field $h$, no matter how strong.

Finally we note from the vanishing of $w(0, h) \equiv \lim _{k \rightarrow 0} w(k, h)=0$ that, if $w(0, h)$ were analytic in $h$, then all derivatives of $w(0, h)$ with respect to $h$ would vanish. In particular the second derivative would vanish, $\left.\frac{\partial^{2} w(0, h)}{\partial h^{2}}\right|_{h=0}=2 D(0)=0$, which states that the gluon propagator $D(k)$ vanishes at $k=0$. Conversely, if $D(0)>0$, then $w(0, h)$ is non-analytic in $h$. Numerical data indicate that $D(0)>0$ in Euclidean dimension $d=3,4,[9,10,11,12,13,14]$, while $D(0)=0$ for $d=2$ 
$[15,9,16]$. Thus non-analyticity of $w(0, h)$ is implied by the lattice data in Euclidean dimension $d=3$ and 4. The vanishing of $D(0)$ in Euclidean dimension $d=2$ is proven in [17].

The situation may be summarized as follows.

Lattice data, taken at $h=0$, indicate that in $d=3$ and 4 Euclidean dimensions, the gluon propagator $D(k)=D(k, h=0)$. is finite in the infrared,

$$
\lim _{k \rightarrow 0} D(k, 0)>0 .
$$

To be consistent with this, lattice data taken at finite $h$ must satisfy

$$
\lim _{k \rightarrow 0} \lim _{h \rightarrow 0} D(k, h)>0
$$

On the other hand, it has been proven [8] that at finite external field, $h>0$, the gluon propagator vanishes in the infrared,

$$
\lim _{k \rightarrow 0} D(k, h)=0 \quad \text { for all } h>0,
$$

and since the limit of 0 is 0 , we have

$$
\lim _{h \rightarrow 0} \lim _{k \rightarrow 0} D(k, h)=0 .
$$

Thus, if we take the lattice data at face value in $d=3$ and 4 dimensions, the order of limits does not commute, and $D(k, h)$ is not analytic in $h$ at $k=0$.

We now turn to our numerical data taken at finite external field strength $h$.

\section{Numerical study of free energy and gluon propagator in an external field}

The free energy is defined by

$$
\exp [W(J)]=\langle\exp [(J, A)]\rangle
$$

The expectation-value is calculated by Monte Carlo simulation, with specifics provided in [7]. Gauge fixing is done by minimization to minimal Landau gauge, so only the inside of the first Gribov region is sampled,

$$
\begin{gathered}
A \in \Omega ; \\
\partial \cdot A=0 ; \quad \text { and } \quad \lambda_{0}(A) \geq 0,
\end{gathered}
$$

where $\lambda_{0}(A)$ is the lowest non-trivial eigenvalue of $M(A)$.

Figure 1 is a log-log plot, of $w(k, h)$ vs $h$ at various fixed $k$. There are two regions separated by a critical value $h_{\mathrm{cr}}$ of the source strength $h$. Straight lines are power laws in $h$, and the slope gives the power. For $h>h_{\mathrm{cr}}$, the slope is 1 , and $w(k, h)$ rises linearly with $h$. This is consistent with the proven bound (3.6)

$$
w(k, h) \leq k h,
$$

and we are seeing the predicted asymptotic linear behavior. At small $h$ the slope is 2, corresponding to a quadratic $h^{2}$ dependence of $w(k, h)$ on $h$. Recall that the free energy $w(k, h)$ is the generating 

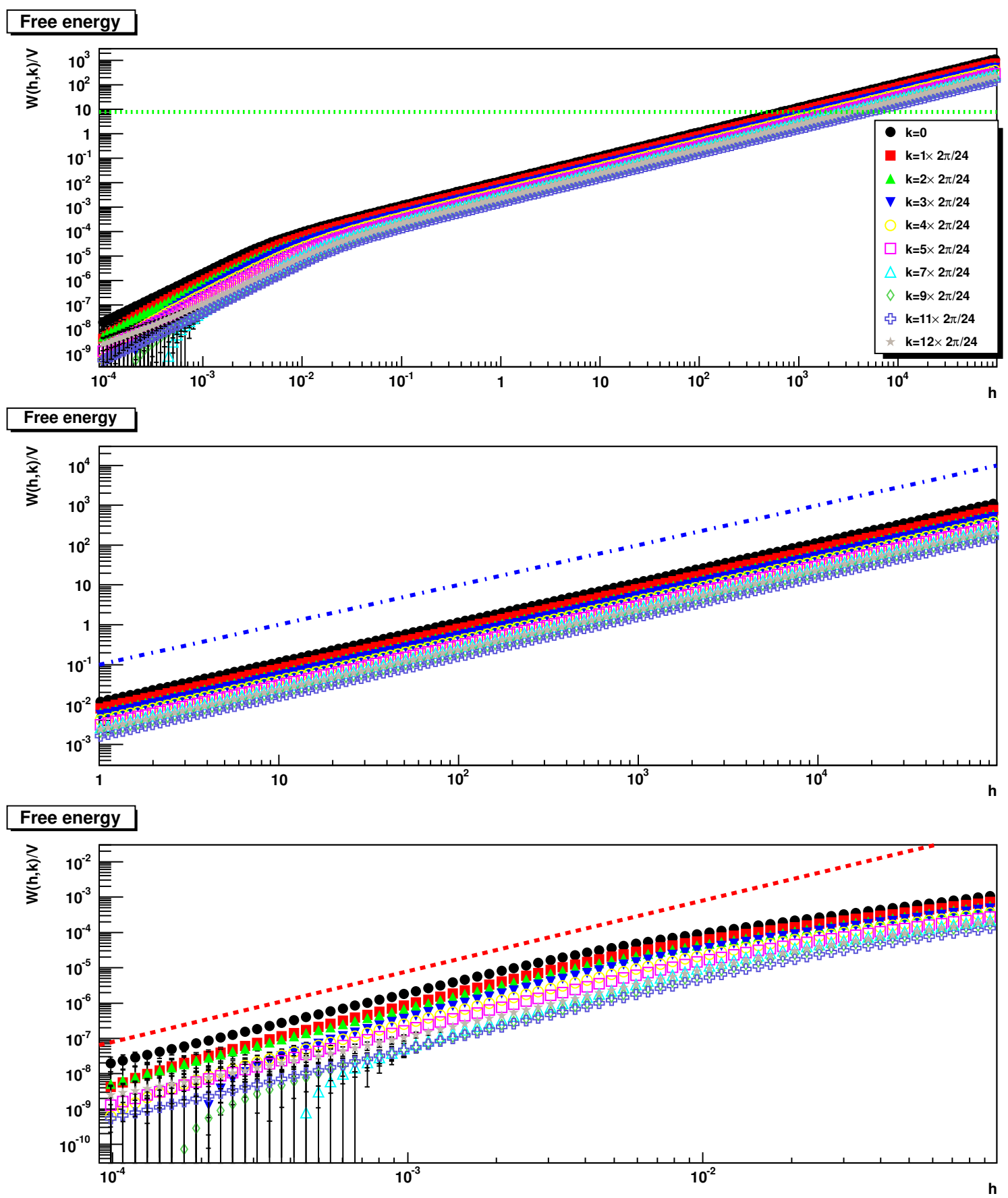

Figure 1: The free energy density $W(h, k) / V$ in lattice units as a function of $h$ for various vales of $k$. The middle and bottom panels show magnifications of the region at large and small $h$, respectively. The reweighting method of calculation cannot be relied upon for values of $h$ above the green dashed line in the top panel. In the middle panel the blue dashed line is linear in $h$, while the red dashed line the bottom panel is proportional to $h^{2}$. The four-dimensional $24^{4}$ lattice has at $\beta=2.221 / a=0.2 \mathrm{fm}$ a physical volume of $(4.8 \mathrm{fm})^{4}$. 

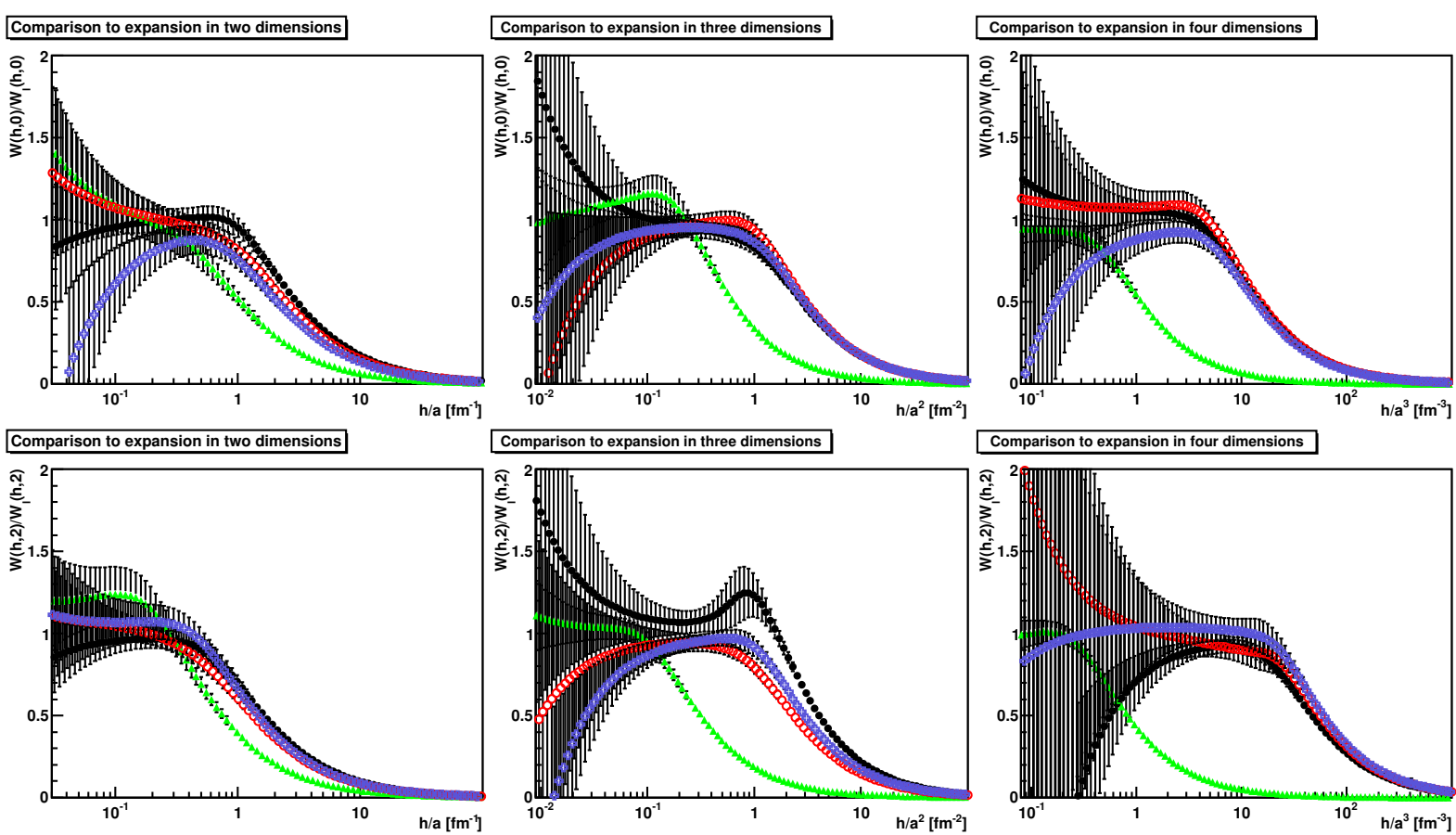

Figure 2: The ratio of the free energy $w(k, h)$ to its leading Taylor coefficient as a function of the source strength $h$. The fixed volume is $(12 \mathrm{fm})^{2}$ in two dimensions, $(3.6 \mathrm{fm})^{3}$ in three dimensions and $(1.2 \mathrm{fm})^{4}$ in four dimensions. Results are shown for $L k / 2 \pi=0,2$ from top to bottom.

functional of the connected correlators. The second derivative of the free energy is the gluon propagator,

$$
\left.\frac{\partial^{2} w(k, h)}{\partial h^{2}}\right|_{h=0}=D(k, 0) .
$$

At low $h$ we are seeing the leading term in the power series expansion

$$
w(k, h)=(1 / 2) D(k, 0) h^{2}+\ldots \text { for } h<h_{\mathrm{cr}},
$$

Thus, the free energy has a very simple behavior. Below $h_{\mathrm{cr}}$ we see the $h^{2}$ term in the power series expansion, and above $h_{\mathrm{cr}}, w(k, h)$ is linear in $h$. This data is also apparent in Figure 2. Below $h_{\text {cr }}$ the ratio $w(k, h) /(1 / 2) D(k, 0) h^{2}$ is unity. A revealing feature of this figure is the green curve that represents a larger volume $V$ than the others. At the larger volume, the region where $a(k, h)$ is constant has moved toward the origin $h=0$.

What is going on? Why is the asymptotic behavior linear?

Let us look at the derivative,

$$
a(k, h)=\frac{\partial w(k, h)}{\partial h}
$$

which is the expectation-value of the $k$-th fourier component of the gluon field $A(x)$ in the presence of the external source $h \cos \left(k x_{1}\right) \delta_{\mu 2} \delta^{a 3}$.

$$
a(k, h)=\langle a(k)\rangle_{h}
$$



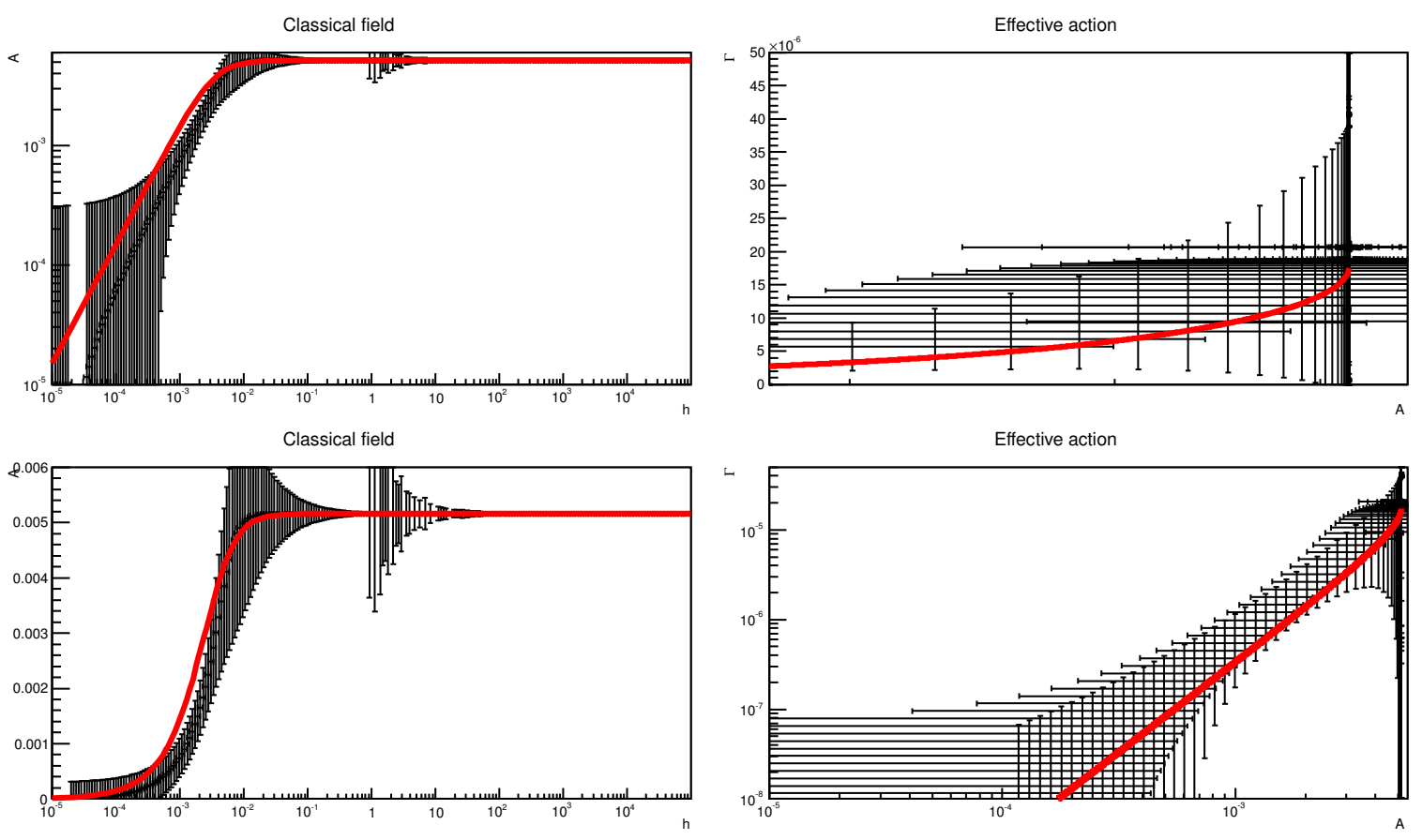

Figure 3: The left panels show the classical field $a(k, h)$ as a function of $h$ in log-log and $\log$ plots. The right panels show the corresponding plots of the quantum effective action $\gamma(k, a)$. The thick red curve represents the fit function given at the end of sect. 4 . Results are for a $(14 \mathrm{fm})^{3}$ lattice with lattice spacing $0.2 \mathrm{fm}$.

The left panels of Fig. 3 show $\log -\log$ and $\log$ plots of $a(k, h)$ as a function of the external field $h$ at fixed sample value $k$. The two regions are again visible. For $h>h_{\mathrm{cr}}, a(k, h)$ is constant, no matter how strong $h$ is, corresponding to,

$$
a(k, h)=c k \text { for } h>h_{\mathrm{cr}} .
$$

At low source strength a reasonable fit is

$$
a(k, h)=D(k, 0) h \text { for } h<h_{\mathrm{cr}} .
$$

Why is $a(k, h)$ constant above a critical field, $h>h_{\mathrm{cr}}$, no matter how strong?

Answer: For large $h$ the external field pushes $a(k, h)$ against the Gribov horizon where it stops of necessity.

The right-hand panels of Fig. 3 show the quantum effective action per unit Euclcidean volume, $\gamma(k, a)$, obtained from $w(k, h)$ by Legendre transform,

$$
\gamma(k, a)=a h-w(k, h) .
$$

An excellent fit to the data is provided by the simple formula,

$$
w(k, h)=\left(m^{2 d}+c^{2} k^{2} h^{2}\right)^{1 / 2}-m^{d},
$$




\section{Example Gribov region}

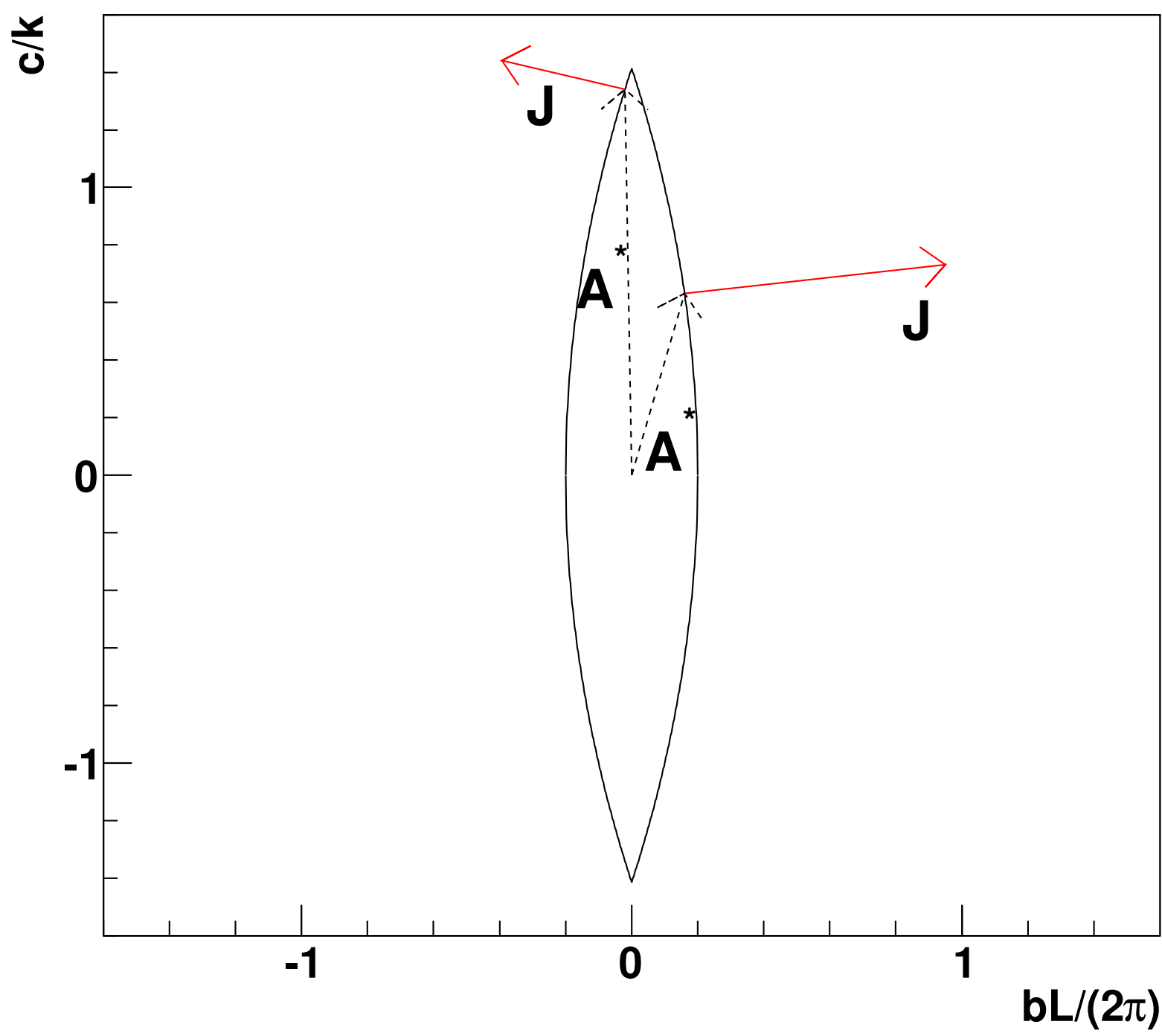

Figure 4: The intersection of the Gribov region $\Omega$ with the 2-plane $P(b, c)$ is contained between the two parabolas. For each $J$ there is a bound on the free energy given by $W(J) \leq\left(J, A^{*}\right)$, where $J$ and $A^{*}=A^{*}(J)$ are illustrated here.

which gives

$$
\begin{gathered}
a(k, h)=\frac{\partial w}{\partial h}=\frac{c^{2} k^{2} h}{\left(m^{2 d}+c^{2} k^{2} h^{2}\right)^{1 / 2}} \\
\gamma(k, a)=m^{d}\left[1-\left(1-\frac{a^{2}}{c^{2} k^{2}}\right)^{1 / 2}\right] .
\end{gathered}
$$

This fit is shown in each panel of Fig. 3 by the thick red line. Note that the quantum effective action 
is undefined beyond the Gribov horizon $a=c k$, and its derivative,

$$
h(k, a)=\frac{\partial \gamma(k, a)}{\partial a}=\frac{m^{d} a / c k}{\left(c^{2} k^{2}-a^{2}\right)^{1 / 2}},
$$

diverges as $a$ approaches the Gribov horizon, $a \rightarrow c k$.

\section{Where is the Gribov horizon?}

We consider configurations in the plane labelled by two parameters, $b$ and $c$,

$$
A_{\mu}^{a}(x ; b, c)=\left[b+c \cos \left(k x_{1}\right)\right] \delta_{\mu 2} \delta^{a 3} .
$$

The Gribov region, given by $\lambda_{0}(A) \geq 0$, is found by calculating the lowest non-trivial eigenvalue $\lambda_{0}(A)$ of the Faddeev-Popov operator $M(A)=-\partial^{2}-A_{\mu} \times \partial_{\mu}$, for $A(b, c)$ of this form, on a Euclidean volume $V=L^{d}$ [7]. The maximum value of $b$ is $b=2 \pi / L$, and the maximum value of $c$ is $c=k / \sqrt{2}$. This calculation is for large volumes $L^{d}$ at fixed momentum $k$, so $2 \pi / L<<k$. The source $J$ is in the direction of the normal to the Gribov horizon,

$$
J_{x}=\frac{\partial \lambda_{0}(A)}{\partial A_{x}}
$$

at $A=A^{*}(J)$, which is found from

$$
J^{a}(x)=-f^{c a b} \psi_{0}^{c *}(x) \psi_{0}^{b}(x) .
$$

Figure 4 illustrates the relation of the source $J$ and the configuration $A^{*}=A^{*}(J)$ which maximizes the inner product $(J, A)$ for fixed $J$ with respect to $A$ in the Gribov region.

\section{Conclusion}

We have understood quite a bit more about how Yang-Mills theory works in the minimal Landau gauge. We have resolved the apparent discrepancies from different approaches to determining the gluon propagator. We have found that they do not disagree, they just take limits in opposite order, and thus obtain consistently different results.

\section{References}

[1] Daniel Zwanziger Phys. Letts. 114B 337 (1981).

[2] T. Maskawa and H. Nakajima Prog. Theor. Phys. 60, 1526 (1978), Prog. Theor. Phys. 63, 642 (1980).

[3] M. Semenov-Tyan-Shanskii and V. Franke, Zap. Nauch. Sem. Leningrad. Otdelleniya Matematicheskogo Instituta in V. A. Steklov, AN SSSR, 120, 159 (1982), (In English translation: New York, Plenum Press 1986).

[4] V. N. Gribov, Nucl. Phys. B 1391 (1978).

[5] A. Maas and T. Mufti, arXiv:1312.4873 [hep-lat]

[6] Daniel Zwanziger, Nucl. Phys. B 209336 (1982). 
[7] A. Maas and D. Zwanziger, arXiv:1309.1957 [hep-lat] and Phys. Rev. D (to be published).

[8] Daniel Zwanziger, Nucl. Phys. B 364, 127 (1991).

[9] A. Cucchieri and T. Mendes, Phys. Rev. Lett. 100241601 (2008) and arXiv:0712.3517 [hep-lat].

[10] A. Cucchieri and T. Mendes, PoS, LAT2007, 297 (2007) and arXiv:0710.0412 [hep-lat].

[11] A. Cucchieri and T. Mendes, arXiv:1001.2584 [hep-lat].

[12] I. L. Bogolubsky, E. M. Ilgenfritz, M. Muller-Preussker, and A. Sternbeck, PoS LAT2007, 290 (2007) and arXiv:0710.1968 [hep-lat].

[13] I. L. Bogolubsky, E. M. Ilgenfritz, M. Muller-Preussker, and A. Sternbeck, Phys. Lett. B676 69 (2009) and arXiv:0901.0736 [hep-lat].

[14] V. Bornyakov, V. Mitrjushkin, and M. Muller-Preussker, Phys. Rev. D81 054503 (2010) and arXiv:0912.4475 [hep-lat].

[15] A. Maas, Phys. Rev. D75 116004 (2007) and arXiv:0704.0722 [hep-lat].

[16] A. Cucchieri and T. Mendes, AIP Conf. Proc. 1343:185 (2011) and arXiv:1101.4779 [hep-lat].

[17] Daniel Zwanziger, Phys. Rev. D 87085039 (2013) and e-print arXiv:1209.1974. 